\title{
Evaluation of PECAM-1 Gene Polymorphism in Patients with Periodontal Disease and Healthy Individuals
}

\author{
Mahdi Kdkhodazadeh, ${ }^{1}$ Mehrdad Hajilooi, ${ }^{2}$ Behzad Houshmand, ${ }^{1}$ \\ Sara Khazaei, ${ }^{3}$ Leila Gholami, ${ }^{3}$ and Sara Alijani ${ }^{4}$ \\ ${ }^{1}$ Periodontics Department, Dental School, Shahid Beheshti University of Medical Sciences, Hamadan, Iran \\ ${ }^{2}$ Immunology Department, Dental School, Hamadan University of Medical Sciences, Hamadan, Iran \\ ${ }^{3}$ Dental School, Hamadan University of Medical Sciences, Hamadan, Iran \\ ${ }^{4}$ Dental School, Shahid Beheshti University of Medical Sciences, Hamadan, Iran \\ Correspondence should be addressed to Behzad Houshmand, houshmandperio@rocketmail.com \\ Received 1 December 2011; Accepted 25 December 2011 \\ Academic Editor: J. H. Jeng
}

Copyright ( $) 2012$ Mahdi Kdkhodazadeh et al. This is an open access article distributed under the Creative Commons Attribution License, which permits unrestricted use, distribution, and reproduction in any medium, provided the original work is properly cited.

Objective. Our aim in this paper was to investigate the possible genetic association between three Ser563Asn, Leu125Val and Arg670Gly polymorphisms of the PECAM-1 gene and periodontitis. Methods. Genomic DNA was isolated from whole blood of 105 periodontal patient ( 52 with chronic periodontitis and 53 with aggressive periodontitis) and 101 healthy individuals. Samples were genotyped and analyzed for the three single-nucleotide polymorphisms (SNPs) of PECAM-1 using polymerase chain reaction with sequence-specific primers (PCR-SSPs). Results. A statistically significant difference was found between the genotypic distribution of the Ser563Asn polymorphism in patients with periodontitis compared to controls $(P=0.02)$. But there were no statistically significant difference between the allele frequencies in the different groups $(P=0.05)$. The other two polymorphisms did not show a statistically significant difference in their allele and genotype frequencies between the groups. There was no statistically significant difference found for any of the polymorphisms allele and genotype distribution in aggressive and chronic periodontitis either. Conclusions. No significant association was found between the polymorphism tested and the subgroups of periodontitis, further research is still necessary to determine whether this polymorphism can be used as a genetic marker of periodontitis.

\section{Introduction}

Genetic associations have been claimed to be effective in predisposition of individuals to periodontitis and have been investigated widely in recent years. Periodontal diseases are a result of destructive inflammatory processes affecting the supporting structures of the teeth, causing resorption of alveolar bone, and formation of periodontal pockets [1]. Not everyone is equally susceptible to periodontitis. The risk for development and/or progression of periodontitis are thought to be determined in part by the host's genotype [2] Recently, there has been increasing interest in identifying allelic variants of genes that can be used in risk assessment of periodontal diseases [3]. Platelet endothelial cell adhesion molecule-1 (PECAM-1/CD31) is a $130-\mathrm{kD}$ vascular cell adhesion and signaling molecule of the immunoglobulin (Ig) superfamily that is expressed on the surface of circulating platelets, monocytes, neutrophils, and selected T-cell subsets. It is also a major constituent of the endothelial cell intercellular junction and plays a role in neutrophil recruitment at inflammatory sites. There is good evidence to suggest that PECAM-1 is a key participant in the adhesion cascade leading to extravasation of leukocytes during the inflammatory process [4]. PECAM-1 is encoded by a $75 \mathrm{~kb}$ gene that resides at the end of the long arm of chromosome 17 [5]. It is a relatively large gene $(110 \mathrm{kbp})$ containing 16 exons encoding the 6 Ig-like extracellular domains (exons 3-8), a transmembrane domain (exon 9), and a relatively long cytoplasmic domain (exons 10-16) [6, 7]. Exons 1 and 2 encode the $5^{\prime}$ untranslated regions and the signal peptide [8].

Several aminoacid polymorphisms have been identified in PECAM-1. These are located in exon 3 codon 80 altering a valine to a methionine (V80M), exon 3 at codon 125 altering a leucine to a valine (L125V), in exon 8 at codon 563 altering 
TABLE 1: Allele and genotype frequencies of the PECAM-1 and Arg670Gly polymorphism in patients with periodontitis and controls.

\begin{tabular}{llcc}
\hline PECAM-1 and Arg670Gly polymorphism & Periodontitis $n=105$ & Controls $n=101$ & $P$ value \\
\hline Allele— $n(\%)$ & & & $99(48.9 \%)$ \\
Arg & $103(49 \%)$ & $103(51 \%)$ & 0.99 \\
Gly & $107(50.9 \%)$ & & \\
Genotype- $n(\%)$ & & $4(4 \%)$ & 0.18 \\
Gly/Gly & $8(7.6 \%)$ & $95(93.9 \%)$ & \\
Gly/Arg & $91(86.7 \%)$ & $2(2 \%)$ & \\
Arg/Arg & $6(5.7 \%)$ & & \\
\hline
\end{tabular}

Values are the numbers (percentages) of patients or controls positive for each allele or genotype. The $P$ values were calculated by the $\chi^{2}$ test from $3 \times 2$ or $2 \times 2$ contingency tables for genotypes and alleles, respectively.

an serine to a asparagine (S563N), and in exon 12 at codon 670 altering an arginine to a glycine (R670G). The latter three polymorphisms are in a strong linkage disequilibrium [9].

These polymorphisms were investigated in the present paper. They have been previously associated with diseases such as ischemic heart diseases, coronary artery disease, stenosis, myocardial infarction (MI), and arthrosclerosis in a number of studies [9-14].

Since these diseases are similar to periodontitis, since they all are inflammatory in nature, and no study has yet been undertaken on the possible association between these polymorphisms and periodontal disease, we evaluated the contribution of them in susceptibility to periodontal diseases.

\section{Material and Methods}

In this case, control studies a total of 206 nonsmoking subjects were recruited. 105 patients with periodontitis (36 females and 17 males; range of age: 15-60 years) consisting of 53 patients with aggressive periodontitis $(\mathrm{AgP}), 52$ patients with chronic periodontitis (CP), and 101 healthy individuals. Periodontitis was diagnosed based on of three clinical parameters, probing depth (PD), clinical attachment loss (CAL), and bleeding on probing index (BPI) and radiographic findings. None of patients had a history of current manifestations of systemic diseases. Patients with severe medical disorders (diabetes mellitus, immunological disorders, hepatitis, HIV infection, and cardiovascular involvement), use of orthodontic appliances, needing pre-medication for dental treatment, chronic usage of anti-inflammatory drugs, present acute necrotizing ulcerative gingivitis, and pregnant or lactating woman were excluded from the study.

The diagnostic criteria of CP were the following: (1) the amount of periodontal destruction was consistent with the presence of local factors such as plaque and calculus; (2) at least two sites had probing depth $-5 \mathrm{~mm}$ and clinical attachment loss $-1 \mathrm{~mm}$ in every quadrant; the number of the teeth with alveolar bone absorption more than $2 / 3$ root length was less than eight. The clinical criteria of $\mathrm{AgP}$ were eight or more teeth, at least 3 of those were other than central incisors or first molars, had a probing depth $-5 \mathrm{~mm}$ and clinical attachment loss $-3 \mathrm{~mm}$. Subjects, even at young ages not reporting familial aggregation of the disease, were not classified as aggressive periodontitis cases and not included in the present paper. In addition, 101 healthy volunteers, without any periodontal disease, systemic inflammatory disease and surgical treatment in the last four weeks were considered as control. The absence of periodontitis was determined according to the following criteria: (1) a minimum of 22 teeth in situ, (2) having no sites with $>3 \mathrm{~mm}$ probing depth, and (3) the absence of clinical attachment loss. None of the control subjects had a history of periodontitis or tooth loss because of pathogenic tooth mobility. Furthermore, there was no bleeding on probing or radiographic evidence of bone loss in these subjects. All subjects signed an informed consent form before enrolment into the study. $10 \mathrm{~mL}$ of venous blood was collected from each subject into tubes containing $50 \mathrm{mM}$ of EDTA. Genomic DNA was isolated from anticoagulated peripheral blood buffy coat using Miller's salting out method [15]. Then the genomic DNA was kept at $-80^{\circ} \mathrm{C}$ until genotyping. The genotyping was performed using PCR-sequence-specific primers (PCR-SSPs) method [16]. Internal control primers were included to control for false negative reactions. The control primers at a $0.2 \mu \mathrm{M}$ concentration $\left(5^{\prime}\right.$-GCC TTC CCA ACC ATT CCC TT and 5'-TCA CGG ATT TCT GTT GTG TTT C-3') was used to amplify a $796 \mathrm{bp}$ segment of HLA-DR4 gene. The PECAM-1 Ser563Asn polymorphism was identified by the sequence-specific forward primers and in combination with the consensus reverse primers $5^{\prime}$-CTCTCCCTCCTGTTCCTTGT- $3^{\prime}$ and 5'CGGTGGATGAGGTCCAGATT-3', respectively. Sequencespecific forward primers in combination with the consensus reverse primers $5^{\prime}$ TAGGTCACAATGACGATGTCA$3^{\prime}$ and $5^{\prime}$-GTGGGAAATTATCCACAGTCC-3', respectively were used to identify the PECAM-1 Arg670Gly polymorphism and the 5'-TGCTGTTCTATAAGGATGACA-3' forward primer and the $5^{\prime}$-GACTCACCTTCCACCAACAC- $3^{\prime}$ common reverse primer were used for investigation of the Leu125Val polymorphism.

Amplification was carried out using a DNA Technology MTC 400 in a total volume of $15 \mu \mathrm{M}$ that contained $100 \mathrm{ng}$ of genomic DNA, $1 \mathrm{mM}$ each allele-specific primer pair, $200 \mu \mathrm{M}$ dNTP, $10 \mathrm{mM}$ Tris- $\mathrm{HCl}(\mathrm{PH}=8.3), 50 \mathrm{mM} \mathrm{KCl}, 1.5 \mathrm{mM}$ $\mathrm{MgCl}_{2}$, and $0.5 \mathrm{IU}$ Taq DNA polymerase. The reaction was carried out as follows; initial denaturation at $94^{\circ} \mathrm{C}$ for $2 \mathrm{~min}$, followed by 30 cycles of amplification at $96^{\circ} \mathrm{C}$ for $20 \mathrm{~S}$ and annealing at $64^{\circ} \mathrm{C}$ for $50 \mathrm{~S}$, with extention for $40 \mathrm{~S}$ at $72^{\circ} \mathrm{C}$, with a final extention for $2 \mathrm{~min}$ at $72^{\circ} \mathrm{C}$. The 
TABLE 2: Allele and genotype frequencies of the PECAM-1 and Arg670Gly polymorphisms in aggressive and chronic periodontitis.

\begin{tabular}{|c|c|c|c|c|c|}
\hline \multicolumn{6}{|c|}{ PECAM-1 and Arg670Gly } \\
\hline & Arg/Arg & Gly/Arg & Gly/Gly & Arg & Gly \\
\hline Aggressive periodontitis & $(5.7 \%)$ & $(88.7 \%)$ & $(5.7 \%)$ & $(50 \%)$ & $(50 \%)$ \\
\hline Chronic periodontitis & $(5.8 \%)$ & $(84.6 \%)$ & $(9.6 \%)$ & $(48 \%)$ & $(51.9 \%)$ \\
\hline
\end{tabular}

No statistical differences were observed at all comparisons $\left(\chi^{2}\right.$ test, $\left.P>0.05\right)$.

TABLE 3: Allele and genotype frequencies of the PECAM-1 and Leu125Val polymorphism in patients with periodontitis and controls.

\begin{tabular}{|c|c|c|c|}
\hline PECAM-1 and Arg670Gly polymorphism & Periodontitis $n=105$ & Controls $n=101$ & $P$ value \\
\hline \multicolumn{4}{|l|}{ Allele- $n(\%)$} \\
\hline Leu & $83(39.5 \%)$ & $75(37.1 \%)$ & \multirow{2}{*}{0.61} \\
\hline Val & $127(60.4 \%)$ & $127(62.8 \%)$ & \\
\hline \multicolumn{4}{|l|}{ Genotype-n $(\%)$} \\
\hline Leu/Leu & $13(12.4 \%)$ & $12(11.9 \%)$ & \multirow{3}{*}{0.81} \\
\hline Leu/Val & $57(54.3 \%)$ & $51(50.5 \%)$ & \\
\hline Val/Val & $35(33.3 \%)$ & $38(37.6)$ & \\
\hline
\end{tabular}

$P$ Value $<0.05$ was considered statistically significant.

amplified PCR products were analyzed by $2 \%$ agarose gel electrophoresis followed by $0.5 \mu \mathrm{g} / \mathrm{mL}$ ethidium bromide staining and ultraviolet visualization. The agarose gels were reported by an investigator unaware of the samples.

Statistics. Allelic and genotypic frequencies were obtained by direct counting. Contingency tables were used with chisquare tests to compare observed genotype frequencies with those expected under Hardy-Weinberg equilibrium. Chisquare and Fisher's exact tests were used to test for differences in genotype and allele distribution between the groups with the help of SPSS version 13 software. The odds ratio (OR) was calculated along with its 95\% CI, and $P$ Value $<0.05$ was considered statistically significant.The study was approved by the Ethics Committee of University. Written informed consents were obtained before entering the study.

\section{Results}

We investigated the genetic variability of the PECAM-1 gene in 101 healthy controls and 105 patients with a mean age of $33.34 \pm 9.8$ and $29.12 \pm 7.71$, respectively.

The results for the Leu125Val and Arg670Gly Allele and genotype frequencies in patients and controls showed no statistically significant difference (Tables 1 and 3).

The allele frequencies of the Ser563Asn polymorphism showed no significant difference between the patients and controls $(P=0.05)$. There was a statistically significant difference in the genotype distribution of this polymorphism. The homozygous genotype combination of Ser/563 was $23.8 \%$ in controls, $13.3 \%$ in patients $(P=0.02)$ and the homozygous/Asn563 was $4 \%$ in controls, $12.4 \%$ in patients $(P=0.02)$ (Table 5).

In addition, as shown in Tables 2, 4, and 6, there was no statistically significant difference found in allele and genotype differences between different forms of periodontitis.

\section{Discussion}

Periodontitis is an inflammatory disorder characterized by connective tissue and alveolar bone destruction. The correlation of known polymorphisms in components of the human immune system with phenotypes for certain patient groups currently appears to provide the most promising application of genetic determinants of periodontitis. In this paper we investigated the three most popular SNPs of the PECAM-1 gene. The Ser563Asn polymorphism codes the extracellular domain of the PECAM-1 molecule. We found no significant allele frequency distribution difference between the patients and controls for this polymorphism $(P=0.05)$, but a statistically significant difference in the genotype distribution of this polymorphism was found $(P=0.02)$. Therefore, we cannot totally rule out the association of this polymorphism with periodontitis, but certainly further investigations are needed. Although such polymorphisms usually do not affect the gene expression, it results in an aminoacid change from Ser to Asn in the functional Ig-like domain of PECAM-1, but its role is not yet fully understood. It has been shown that the nutrophil specific antigen (CD177) is involved in transmigration of PMN via their attachment to this domain [17].

Some studies have found relationships between this polymorphism and diseases such as stenosis, myocardial infarction (MI), and arthrosclerosis $[10,11,13,14]$. But to our knowledge, there have been no similar studies on the association of this polymorphism and periodontitis to compare our results with.

PECAM-1 creates its hemophilic bonds through its first or second Ig-like (d1/2) extracellular domain [18, 19], and these hemophilic bonds have an important role in interendothelial cell bindings [20] and transendothelial migration of monocytes [21, 22].

Since the Leu125Val $(C+373 G)$ polymorphism is in the first and second domain of PECAM-1 and the importance of 
TABLE 4: Allele and genotype frequencies of the PECAM-1 and Leu125Val polymorphisms in aggressive and chronic periodontitis.

\begin{tabular}{lccccc}
\hline & \multicolumn{3}{c}{ PECAM-1 and Arg670Gly } & & Val. \\
\hline Aggressive periodontitis & Leu/Leu & Leu/Val & Val/Val & Leu & $(62.2 \%)$ \\
Chronic periodontitis & $(11.3 \%)$ & $(52.8 \%)$ & $(35.8 \%)$ & $(37.7 \%)$ & $(58.6 \%)$ \\
\hline
\end{tabular}

No statistical differences were observed at all comparisons ( $\chi^{2}$ test, $\left.P>0.05\right)$.

TABLE 5: Allele and genotype frequencies of the PECAM-1 and Ser563Asn polymorphism in patients with periodontitis and controls.

\begin{tabular}{|c|c|c|c|}
\hline PECAM-1 and Ser563Asn polymorphism & Periodontitis $n=105$ & Controls $n=101$ & $P$ value \\
\hline \multicolumn{4}{|l|}{ Allele- $n(\%)$} \\
\hline Ser & $106(50.4 \%)$ & $121(60 \%)$ & \multirow{2}{*}{0.05} \\
\hline Asn & $104(49.5 \%)$ & $81(40 \%)$ & \\
\hline \multicolumn{4}{|l|}{ Genotype-n $(\%)$} \\
\hline Ser/Ser & $14(13.3 \%)$ & $24(23.8 \%)$ & \multirow{3}{*}{0.02} \\
\hline Ser/Asn & $78(74.3 \%)$ & $73(72.3 \%)$ & \\
\hline Asn/Asn & $13(12.4 \%)$ & $4(4 \%)$ & \\
\hline
\end{tabular}

$P$ Value $<0.05$ was considered statistically significant.

TABLE 6: Allele and genotype frequencies of the PECAM-1 and Ser563Asn polymorphisms in aggressive and chronic periodontitis.

\begin{tabular}{|c|c|c|c|c|c|}
\hline \multicolumn{6}{|c|}{ PECAM-1 and Arg670Gly } \\
\hline & Ser/Ser & Ser/Asn & Asn/Asn & Ser & Asn \\
\hline Aggressive periodontitis & $(13.2 \%)$ & $(73.6 \%)$ & $(13.2 \%)$ & $(50 \%)$ & $(50 \%)$ \\
\hline Chronic periodontitis & $(13.5 \%)$ & $(75 \%)$ & $(11.5 \%)$ & $(50.9 \%)$ & $(49 \%)$ \\
\hline
\end{tabular}

No statistical differences were observed at all comparisons $\left(\chi^{2}\right.$ test, $\left.P>0.05\right)$.

this domain and also previous findings showing the association of this polymorphism with diseases such as coronary artery disease, atherosclerosis, and ischemic heart diseases; $[9,14]$ we decided to investigate its possible association with periodontitis.

The results of our paper showed no statistically significant association between allele and genotype frequency in periodontal patients and controls $(P=0.61$ and $P=0.81)$. We found no similar study to compare our results.

PECAM-1 appears to interact both with it (hemophilic adhesion) and with non-PECAM-1 ligands (heterophilic adhesion).

The cytoplasmic domin of this molecule has an important role in regulating its cellular adhesion behavior and is coded by exons 10-16 [18].

Although the exact role of the exon 12 cytoplasmic domin is not clear yet, the Arg670Gly polymorphism in this exon has been found to be related to diseases such as ischemic heart diseases, MI and Stenosis [10, 12].

Considering the similar immunologic and inflammatory basis of these diseases and periodontitis, we decided to evaluate the possible role of this polymorphism in periodontitis.

However, we found no statistically significant association between the different genotype and allele frequency distribution of this polymorphism and periodontitis.

Taken together, this paper showed no statistically significant relation between these polymorphisms and periodontitis, but further studies are still recommended.

\section{Conclusion}

No significant association was found between the polymorphism tested and the subgroups of periodontitis, further research is still necessary to determine whether this polymorphism can be used as a genetic marker of periodontitis.

\section{References}

[1] E. Hannigan, D. P. O’Connell, A. Hannigan, and L. A. Buckley, "Soluble cell adhesion molecules in gingival cervicular fluid in peridontal health and disease," Journal of Periodontology, vol. 75, pp. 546-550, 2004.

[2] B. S. Michalowicz, "Genetic and heritable risk factors in periodontal disease," Journal of Periodontology, vol. 65, no. 5, pp. 479-488, 1994.

[3] D. F. Kinane and T. C. Hart, "Genes and gene polymorphisms associated with periodontal disease," Critical Reviews in Oral Biology and Medicine, vol. 14, no. 6, pp. 430-449, 2003.

[4] R. J. Gumina, N. E. Kirschbaum, P. N. Rao, P. VanTuinen, and P. J. Newman, "The human PECAM1 gene maps to $17 \mathrm{q} 23$," Genomics, vol. 34, no. 2, pp. 229-232, 1996.

[5] J. Dangerfield, K. Y. Larbi, M. T. Huang, A. Dewar, and S. Nourshargh, "PECAM-1 (CD31) homophilic interaction upregulates $\alpha 6 \beta 1$ on transmigrated neutrophils in vivo and plays a functional role in the ability of $\alpha 6$ integrins to mediate leukocyte migration through the perivascular basement membrane," Journal of Experimental Medicine, vol. 196, no. 9, pp. 1201-1211, 2002.

[6] P. J. Newman, M. C. Berndt, J. Gorski et al., "PECAM-1 (CD31) cloning and relation to adhesion molecules of the 
immunoglobulin gene super family," Science, vol. 247, pp. 1219-1222, 1990.

[7] Y. Xie and W. A. Muller, "Molecular cloning and adhesive properties of murine platelet/endothelial cell adhesion molecule 1," Proceedings of the National Academy of Sciences of the United States of America, vol. 90, no. 12, pp. 5569-5573, 1993.

[8] Y. Wang, X. Su, C. M. Sorenson, and N. Sheibani, "Tissuespecific distributions of alternatively spliced human PECAM-1 isoforms," American Journal of Physiology, Heart and Circulatory Physiology, vol. 284, no. 3, pp. H1008-H1017, 2003.

[9] L. Fang, H. Wei, S. H. Chowdhury et al., "Association of Leu125Val polymorphism of platelet endothelial cell adhesion molecule-1 (PECAM-1) gene \& soluble level of PECAM-1 with coronary artery disease in Asian Indians," Indian Journal of Medical Research, vol. 121, no. 2, pp. 92-99, 2005.

[10] F. Liao, H. K. Huynh, A. Eiroa, T. Greene, E. Polizzi, and W. A. Muller, "Migration of monocytes across endothelium and passage through extracellular matrix involve separate molecular domains of PECAM-1," Journal of Experimental Medicine, vol. 182, no. 5, pp. 1337-1343, 1995.

[11] M. A. Elrayess, K. E. Webb, G. J. Bellingan et al., "R643G polymorphism in PECAM-1 influences transendothelial migration of monocytes and is associated with progression of CHD and CHD events," Atherosclerosis, vol. 177, no. 1, pp. 127-135, 2004.

[12] T. Sasaoka, A. Kimura, S. Hohta, N. Fukuda, T. Kurosawa, and T. Izumi, "Polymorphisms in the platelet-endothelial cell adhesion molecule-1 (PECAM-1) gene, Asn563Ser and Gly670Arg, associated with myocardial infarction in the Japanese," Annals of the New York Academy of Sciences, vol. 947, pp. 259-269, 2001.

[13] Y. Yang, L. Cheng, R. Nsenga, M. He, Z. Chang, and T. Wu, "Association of $\mathrm{G}+1688$ A polymorphism of platelet endothelial cell adhesion molecule-1 gene with myocardial infarction in the Chinese Han population," Journal of Huazhong University of Science and Technology, vol. 27, no. 5, pp. 520-523, 2007.

[14] J. Auer, T. Weber, R. Berent, E. Lassnig, G. Lamm, and B. Eber, "Genetic polymorphisms in cytokine and adhesion molecule genes in coronary artery disease," American Journal of PharmacoGenomics, vol. 3, no. 5, pp. 317-328, 2003.

[15] S. A. Miller, D. D. Dykes, and H. F. Polesky, "A simple salting out procedure for extracting DNA from human nucleated cells," Nucleic Acids Research, vol. 16, no. 3, p. 1215, 1988.

[16] S. Borozdenkova, J. Smith, S. Marshall, M. Yacoub, and M. Rose, "Identification of ICAM-1 polymorphism that is associated with protection from transplant associated vasculopathy after cardiac transplantation," Human Immunology, vol. 62, no. 3, pp. 247-255, 2001.

[17] U. J. H. Sachs, C. L. Andrei-Selmer, A. Maniar et al., "The neutrophil-specific antigen CD177 is a counter-receptor for platelet endothelial cell adhesion molecule-1 (CD31)," Journal of Biological Chemistry, vol. 282, no. 32, pp. 23603-23612, 2007.

[18] W. A. Muller, S. A. Weigl, X. Deng, and D. M. Phillips, "PECAM-1 is required for transendothelial migration of leukocytes," Journal of Experimental Medicine, vol. 178, no. 2, pp. 449-460, 1993.

[19] J. Sun, J. Williams, H. C. Yan, K. M. Amin, S. M. Albelda, and H. M. DeLisser, "Platelet endothelial cell adhesion molecule-1 (PECAM-1) homophilic adhesion is mediated by immunoglobulin-like domains 1 and 2 and depends on the cytoplasmic domain and the level of surface expression,"
Journal of Biological Chemistry, vol. 271, no. 31, pp. 1856118570, 1996.

[20] I. N. Bird, V. Taylor, J. P. Newton et al., "Homophilic PECAM1 (CD31) interactions prevent endothelial cell apoptosis but do not support cell spreading or migration," Journal of Cell Science, vol. 112, no. 12, pp. 1989-1997, 1999.

[21] W. J. Laesche, "Chemotheraphy of dental plaque infection," Oral Science Reviews, vol. 9, pp. 65-107, 1967.

[22] W. E. C. Moore and L. V. Holdeman, "Special problems associated with the isolation and identification of intestinal bacteria in fecal flora studies," American Journal of Clinical Nutrition, vol. 27, no. 12, pp. 1450-1455, 1974. 


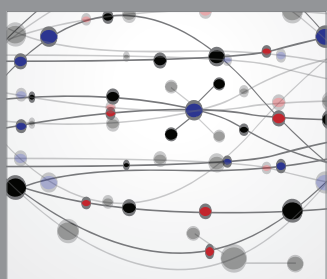

The Scientific World Journal
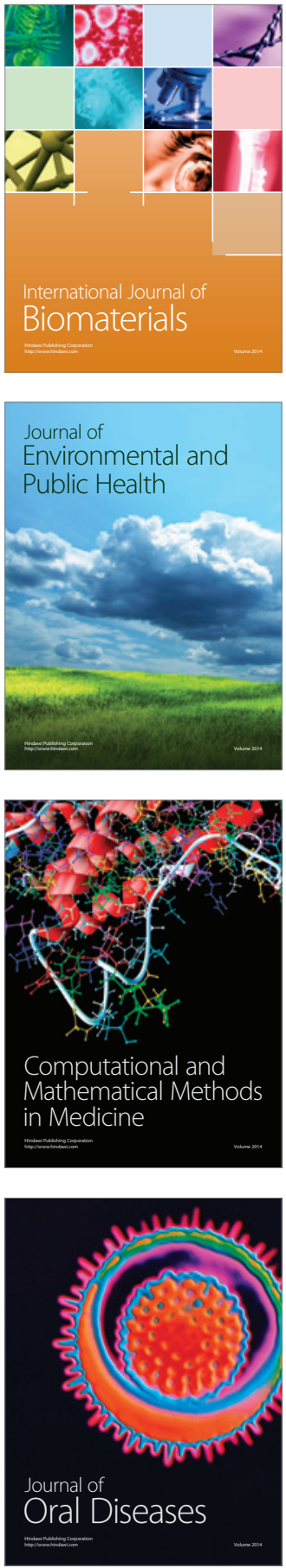
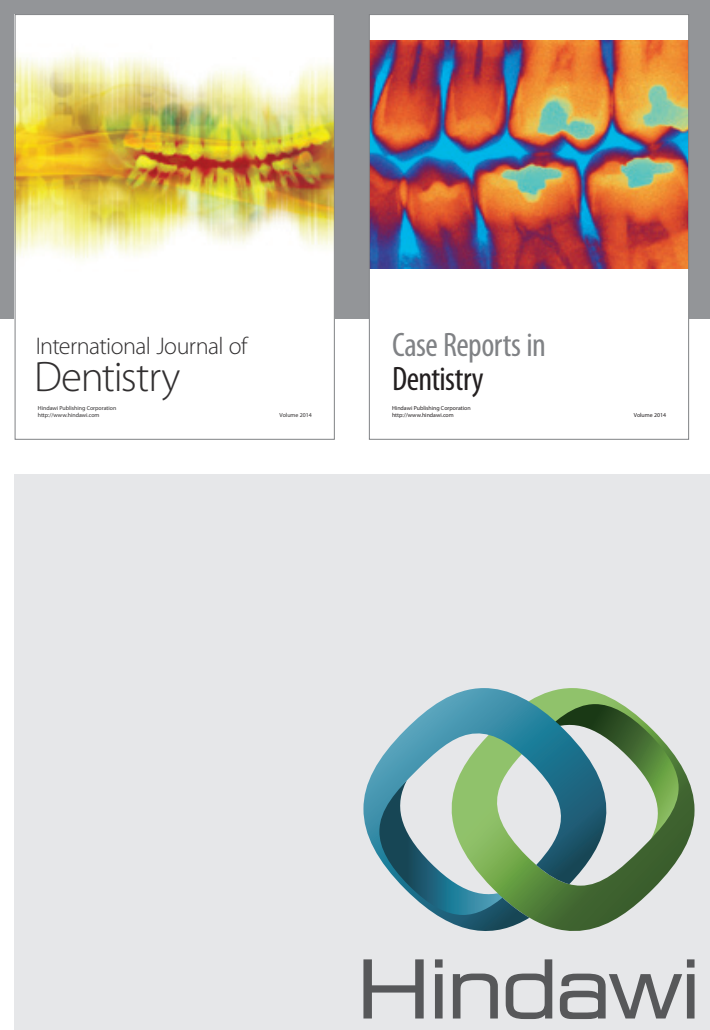

Submit your manuscripts at

http://www.hindawi.com
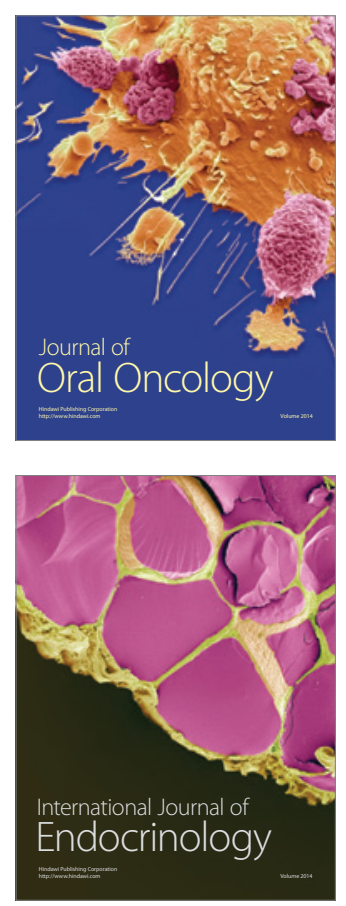
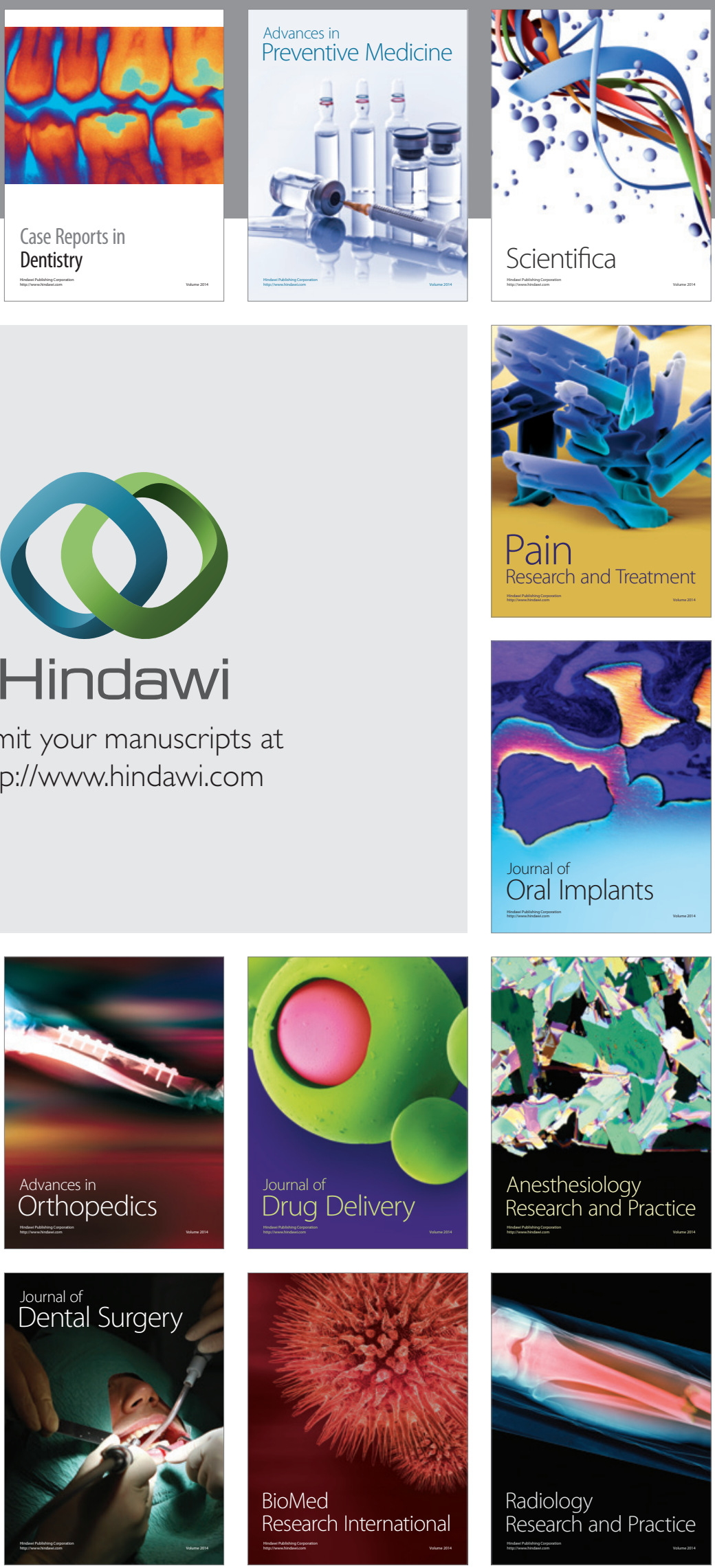